NOTA

\title{
Nuevos registros de plantas comidas por el tapir, Tapirus bairdii (Perissodactyla: Tapiridae), en el Parque Nacional Braulio Carrillo, Costa Rica
}

José Pablo Carvajal $^{1}$ (D), Pablo Marín Pacheco ${ }^{1}$ (D) , Alexander Espinoza Chacón ${ }^{1}$ (D), Franco Rohrmoser Steinvorth ${ }^{1}$ (D) \& Christian Pérez Pérez ${ }^{2}$ (iD)

1. Universidad Técnica Nacional, Ingeniería en Manejo Forestal y Vida Silvestre, Sede Atenas, Costa Rica; jcarvajals@utn.ac.cr, pablomarin95@hotmail.com, arespinozac@est.utn.ac.cr;,frs990@gmail.com,

2. Universidad Nacional, Conservación y Manejo de Áreas Protegidas, Instituto de Conservación y Manejo de Vida Silvestre, SINAC, Costa Rica; cristian.perez@sinac.go.cr

Recibido 13-II-2020 • Corregido 21-IV-2020 • Aceptado 23-IV-2020

DOI: https://doi.org/10.22458/ urj.v12i1.2771

\begin{abstract}
New records of plants eaten by the tapir, Tapirus bairdii (Perissodactyla: Tapiridae) in Braulio Carrillo National Park, Costa Rica". We report Tapirus bairdii eating Schefflera rodriguesiana and Gunnera insignis in Volcán Barva, Costa Rica.
\end{abstract}

Key words: Tapirus bairdii, Tapir, herbivory, Volcano Barva, Schefflera rodriguesiana, Gunnera insignis.
RESUMEN. Informamos a Tapirus bairdii consumiendo Schefflera rodriguesiana y Gunnera insignis en el Volcán Barva, Costa Rica.

Palaras clave: Tapirus bairdii, danta, herbívoro, Volcán Barva, Schefflera rodriguesiana, Gunnera insignis.

El tapir centroamericano, llamado danta en Costa Rica, destaca por ser el mamífero terrestre de mayor peso del Neotrópico, logrando alcanzar 150 a 300kg (Naranjo \& Aldán, 1998). Esta especie se distribuye desde el centro y sur de México hasta el noroeste de Colombia y Ecuador. Por consecuente, es la única especie de Tapirus que habita los bosques de Mesoamérica. (Carrillo, Wong, \& Sáenz, 2002; Naranjo, 2009; Lavariega, Briones-Salas, \& Rodríguez, 2013, Schank et al., 2020).

La danta dispersa semillas y forma claros tras su paso, lo cual permite la sobrevivencia de plántulas y así mantiene mayor heterogeneidad espacial. Por tales razones, se considera la "jardinera" del bosque, ya que es influyente en el mantenimiento y dinámica de ecosistemas ( Janzen, 1982; Semarnat, 2009; Botello, Sánchez-Hernández, Hernández, Reyes-Chávez, \& SánchezCordero, 2014).

La danta dispone una alimentación herbívora, y la base de su dieta consiste en el consumo de hojas, tallos, frutos, semillas digeridas, cortezas y flores (Foester, 1998; Wainwrigth, 2007). Las dantas presentan una preferencia por sitios con plantas de rápido crecimiento y de vida corta, ya que esta composición vegetal, es creído tener altos valores nutricionales y menos compuestos secundarios tóxicos (Naranjo \& Aldán, 1998; Wainwrigth, 2007). Foester y Vaughan (2002) investigaron la alimentación de una danta hembra y se determinó que se alimentan de 126 especies de plantas, divididos entre bejucos, árboles, arbustos y hierbas, la dieta consistió en $67,0 \%$ hojas, $18,6 \%$ fruta, $11,7 \%$ tallos, $2,1 \%$ corteza, y $0,1 \%$ flores. Igualmente, otros estudios en Costa Rica y 
Panamá sobre la dieta de la danta basados en la recolección de excretas, han registrado que las familias vegetales con mayor frecuencia son: Moraceae, Rubiaceae, Fabaceae, Asteraceae, Ericaceae y Poaceae, destacando las especies vegetales: Chusquea subtessellata, Hymenaea courbaril, Crescentia alata, Ochroma pyramidade, Alibertia edulis, Guazuma ulmifolia, siendo estas aprovechadas principalmente por follaje y frutos (Wainwrigth, 2007; Semarnat, 2009).

Esta nota hace referencia a nuevos registros en la dieta de la danta (Tapirus bairdii), las cuales se determinaron por observación directa y con la colocación de cámaras trampa en el Parque Nacional Braulio Carrillo, Sector Volcán Barva, Área de Conservación Central, Costa Rica. El 11 de octubre del 2016, al ser las 12:49am, se observa un individuo de Tapirus bairdii comiendo de la corteza de la especie arbórea Schefflera rodriguesiana (Araliaceae), en un sitio $10.136766 \mathrm{~N}$, 84.109616W, (Fig. 1, Fig. 2), a los 2 795m de elevación. Observamos numerosos árboles de $S$. rodriguesiana con rasgos de mordeduras sobre su corteza, sugiriendo que por lo menos una danta estaba comiendo esta especie más de una vez.

Igualmente, el 16 de octubre, entre las coordenadas $10.133204 \mathrm{~N}, 84.121180$ "W, a los 2 692m de elevación, sobre las 6:16am, se fotografía un individuo alimentándose de Gunnera insignis (Fig. 3). Consideramos necesario por lo anteriormente expuesto dar a conocer estas especies de flora dentro de los registros alimenticios de la danta, para que sean tomados en cuenta por expertos en fauna silvestre, dado que en país existen centros de rescate, que podrían utilizar estas especies tanto en la dieta como en la ambientación de los recintos.

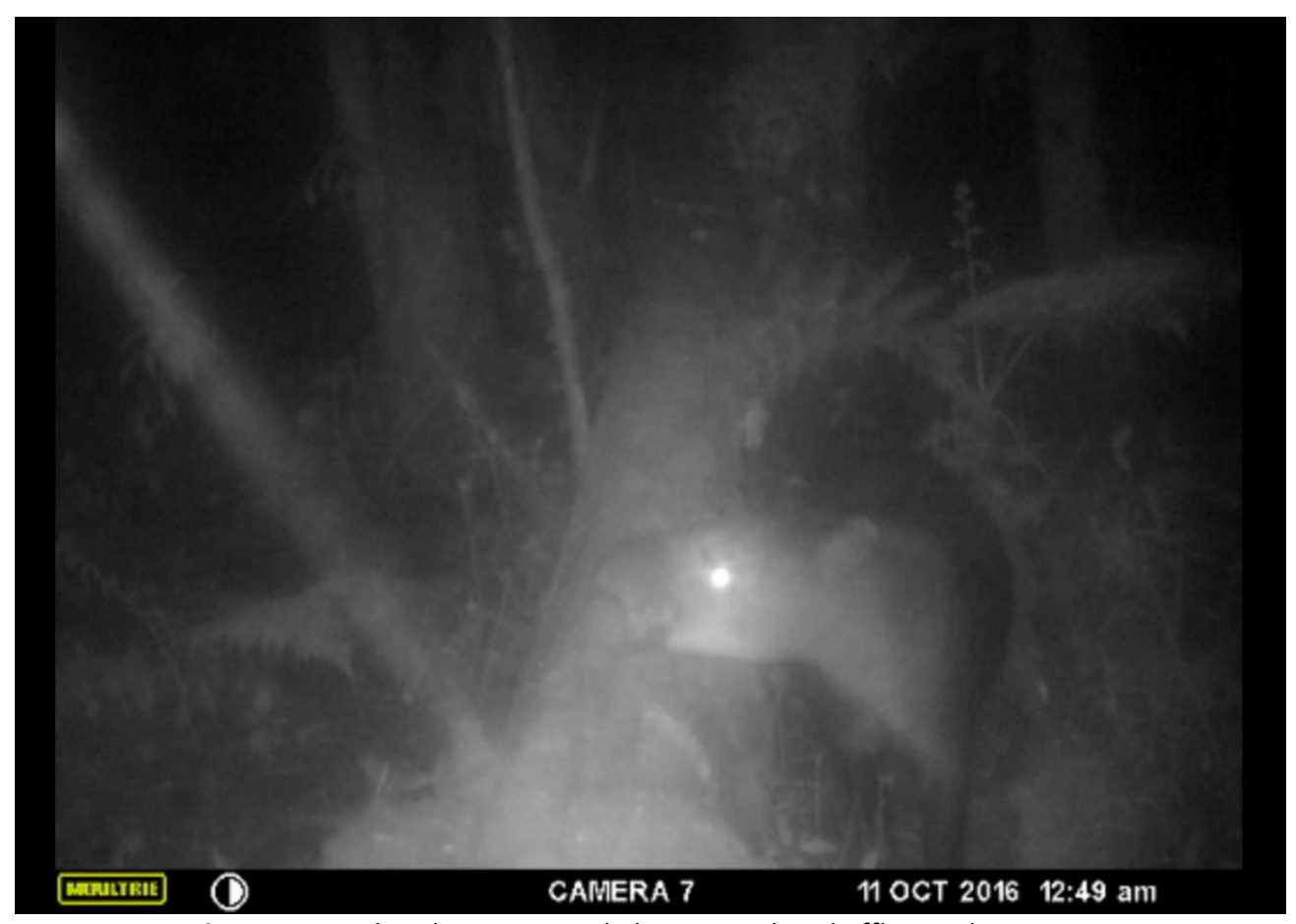

Fig. 1. Tapirus bairdii consumiendo la corteza de Schefflera rodriguesiana. 


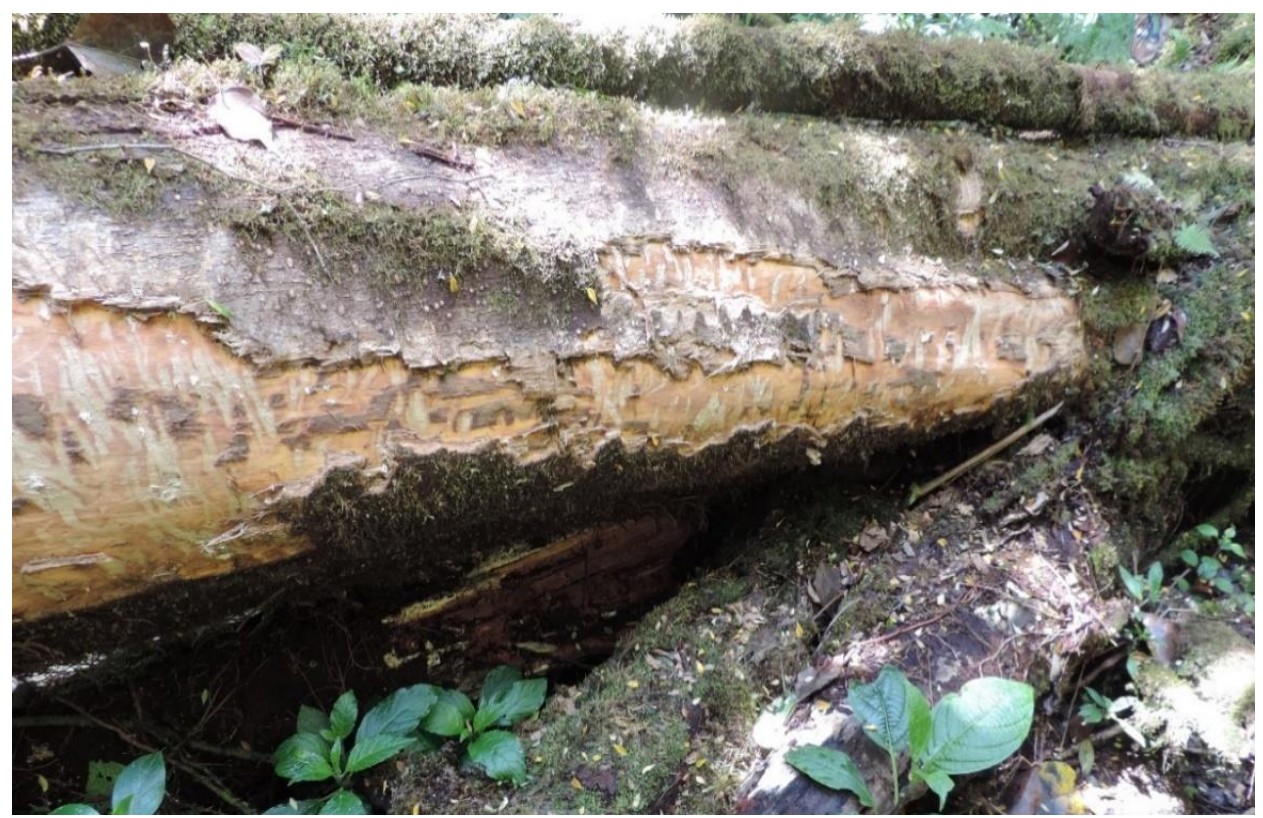

Fig. 2. Rastros de mordeduras de Tapirus bairdii a la corteza arbórea de Schefflera rodriguesiana.

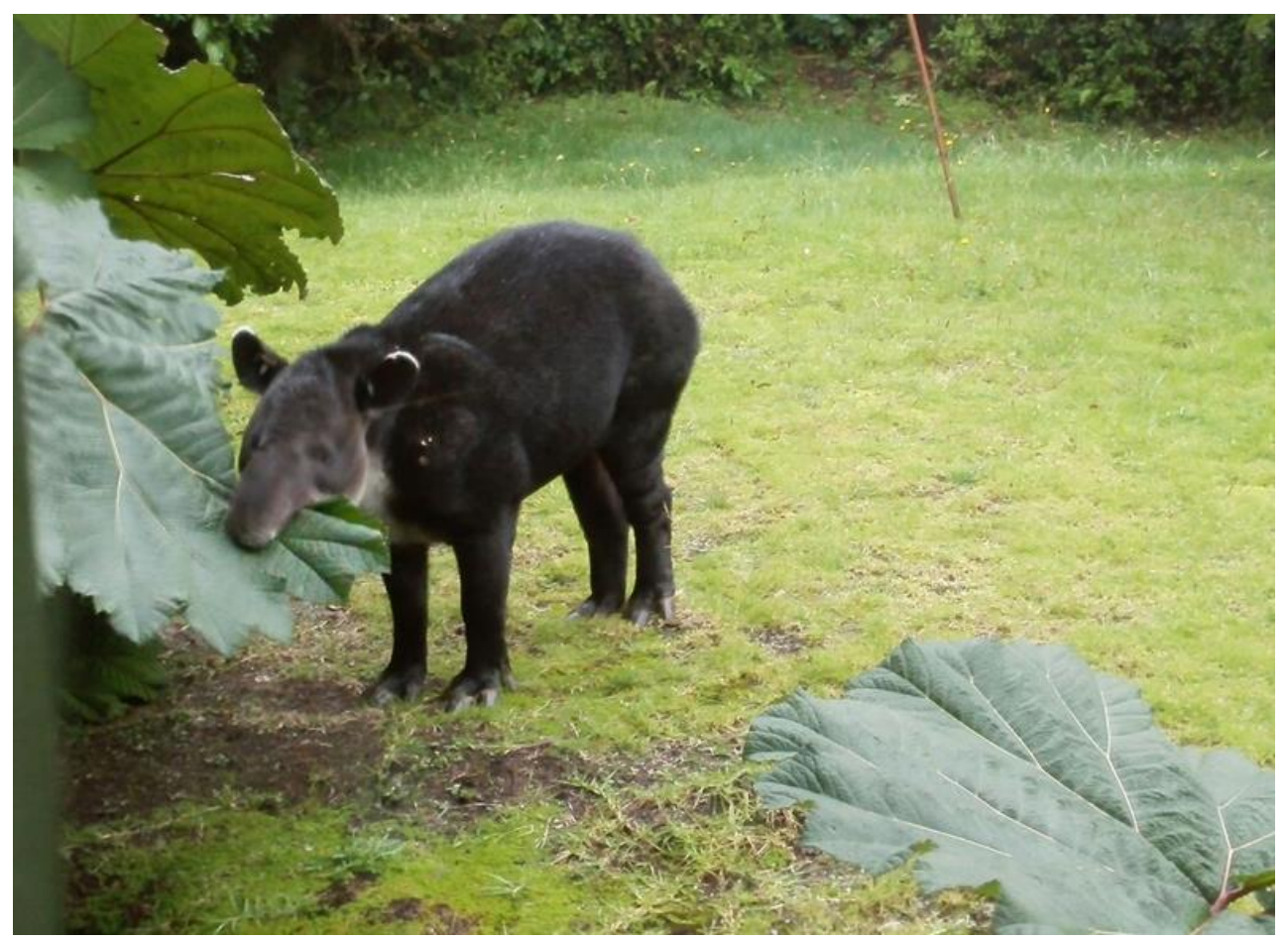

Fig. 3. Tapirus bairdii consumiento las hojas de Gunnera insignis.

Ética, conflicto de intereses y declaración de financiamiento: Los autores declaran haber cumplido con todos los requisitos éticos y legales pertinentes, tanto durante el estudio como en el manuscrito; que no hay conflictos de interés de ningún tipo, y que todas las fuentes financieras se detallan plena y claramente en la sección de agradecimientos. Asimismo, están de acuerdo con la versión editada final del documento. El respectivo documento legal firmado se encuentra en los archivos de la revista. 


\section{REFERENCIAS}

Botello, F., Sánchez-Hernández, J., Hernández, O., Reyes-Chávez, D., \& Sánchez-Cordero, V. (2014). Registros notables del tapir centroamericano (Tapirus bairdii) en la Sierra Mixe, Oaxaca, México. Revista Mexicana de Biodiversidad, 85, 995-999. DOI: 10.7550/rmb.41024

Carrillo, E., Wong, G., \& Sáenz, J. (2002). Mamíferos de Costa Rica. Santo Domingo, Heredia, Costa Rica: Instituto Nacional de Biodiversidad (INBio).

Foerster, C., \& Vaughan, C. (2002). Home range, habitat use and activity of Baird's tapir in Costa Rica. Biotropica, 34(3), 423-437.

Foerster, C. R. (1998). Ecología de la danta Centroamericana Tapirus bairdii en un bosque húmedo tropical de Costa Rica. (Tesis de maestría). Universidad Nacional, Heredia, Costa Rica.

Janzen, D. H. (1982). Seeds in tapir dung in Santa Rosa National Park, Costa Rica. Brenesia, 19/20, 129-135.

Lavariega, M. C., Briones-Salas, M., \& Rodríguez, C. (2013). Registro del tapir centroamericano (Tapirus bairdii) con cámaras-trampa en la sierra Madre de Oaxaca, México. Revista Mexicana de Biodiversidad, 84, 1007-1011. DOI: $10.7550 / \mathrm{rmb} .32857$

Naranjo, P., \& Aldán, C. (1998). Ecología del tapir (Tapirus bairdii) en la Reserva de la Biosfera La Sepultura, Chiapas, México Acta Zoológica Mexicana, 73, 111-125.

Naranjo, E. (2009). Ecology and conservation of Baird's tapir in Mexico. Tropical Conservation Science, 2,140-158. DOI: $10.1177 / 1940082918795558$

Semarnat. (2009). Programa de acción para la conservación de la especie: tapir centroamericano (Tapirus bairdii). Recuperado de https://www.gob.mx/cms/uploads/attachment/file/350217/PACE_Tapir_2009.pdf.

Schank, C., Cove, M., Arima, E., Brandt, L., Brenes-Mora, E., Carver, A.,Díaz-Pulido, A., Estrada, N.,Foster, R., GodínezGómez, O., Harmsen, B., Jordan, C., Keitt, T., Kelly, M., Méndez, J., Mendoza, E., Meyer, N., PozoMontuy, G., Naranjo, E., \& Miller, J. (2020). Population status, connectivity, and conservation action for the endangered Baird's Tapir. Biological Conservation, 245, 108501. DOI: 10.1016/j.biocon.2020.108501.

Wainwrigth, M. (2007). The Mammals of Costa Rica: A Natural History and Field Guide. New York, Estados Unidos: Zona Tropical. 